\title{
A Juvenile Case of Pulmonary Lymphangitic Carcinomatosis Caused by Sigmoid Colon Cancer with a Component of Micropapillary Carcinoma
}

\author{
Kohei Otsubo ${ }^{1}$, Naoki Kubo ${ }^{2}$, Nobutaka Nakashima ${ }^{3}$, Miiru Izumi ${ }^{1}$, \\ Mari Nakamori ${ }^{4}$ and Hiroshi Koto ${ }^{3}$
}

\begin{abstract}
Micropapillary carcinoma is known to be associated with a poor prognosis and high propensity for lymphovascular invasion and lymph node metastasis. Case reports on colorectal micropapillary carcinoma are relatively rare. We report here a 26-year-old woman who had sigmoid colon cancer with a micropapillary component. We made the diagnosis of pulmonary lymphangitic carcinomatosis but could not identify the primary lesion. We gave her chemotherapy as an occult primary cancer. But her respiratory condition did not improve and she died of respiratory failure. Autopsy was performed after her death. The final diagnosis was pulmonary lymphangitic carcinomatosis and multiple lymph node metastases of sigmoid colon cancer with a component of micropapillary carcinoma.
\end{abstract}

Key words: colon cancer, lymphangitis, metastasis, respiratory failure, autopsy

(Intern Med 50: 2361-2365, 2011)

(DOI: 10.2169/internalmedicine.50.5170)

\section{Introduction}

Micropapillary carcinoma (MC) is characterized by a small tight cluster of neoplastic cells floating in clear spaces resembling lymphatic channels and it is known to be associated with a poor prognosis and high propensity for lymphovascular invasion and lymph node metastasis (1). There are several reports on the MC cases of the breast, urinary bladder, lung, ovary and salivary gland (2-6). However, the reports on colorectal MC cases are relatively rare. We encountered a young patient who had sigmoid colon cancer with a micropapillary component and pulmonary lymphangitic carcinomatosis. In this report, we describe her clinical course and autopsy findings.

\section{Case Report}

In mid-April 2009, a previously healthy 26-year-old woman was admitted to Kyushu Central Hospital for further evaluation and treatment of respiratory failure. She had been suffering from dry cough and dyspnea on exertion for one month. A day before admission to our hospital, she was admitted to another hospital, where diffuse ground-glass opacity on chest radiograph was pointed out. She was diagnosed with mycoplasma pneumonia and treated with antimicrobial agents (ciprofloxacin and azithromycin). Her respiratory status, however, progressively deteriorated and then she was transferred to our hospital on the following day.

On presentation, her temperature was $37.1^{\circ} \mathrm{C}$, pulse 132 beats per mimute, blood pressure $146 / 97 \mathrm{mmHg}$, and respiratory rate 30 breaths per mimute. Oxygen saturation was 94\% with supplemental oxygen (10 litters via a nonrebreathing face mask). Crackles were present in bilateral chest. An enlarged right supraclavicular lymph node was felt.

Blood test results showed: white blood cell count 17.1× $10^{3} / \mathrm{\mu L}$ (neutrophils $82.8 \%$ ), hemoglobin $13.9 \mathrm{~g} / \mathrm{dL}$, platelets

\footnotetext{
${ }^{1}$ Research Institute for Diseases of the Chest, Graduate School of Medical Sciences, Kyushu University, Japan, ${ }^{2}$ Department of Respiratory Medicine, National Hospital Organization Fukuoka Higashi Medical Center, Japan, ${ }^{3}$ Department of Respiratory Medicine, Kyushu Central Hospital, Japan and ${ }^{4}$ Department of Pathology, Kyushu Central Hospital, Japan

Received for publication January 17, 2011; Accepted for publication July 7, 2011

Correspondence to Dr. Kohei Otsubo, ootsubo@kokyu.med.kyushu-u.ac.jp
} 


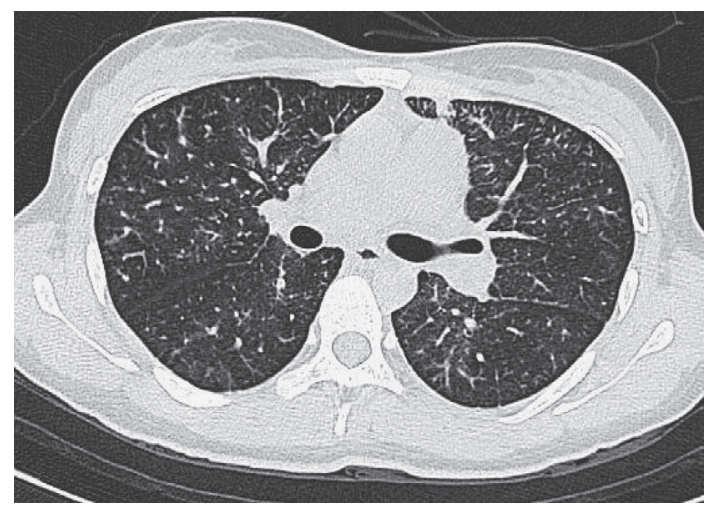

Figure 1. Thoracic HRCT showed diffuse peribronchovascular interstitial thickening, nodular interlobular septal thickening, and nodular thickening of fissures.

$19.3 \times 10^{4} / \mu \mathrm{L}$, and C-reactive protein (CRP) $3.26 \mathrm{mg} / \mathrm{dL}$. Thoracic high resolution computed tomography (HRCT) showed diffuse peribronchovascular interstitial thickening, nodular interlobular septal thickening, and nodular thickening of fissures (Fig. 1). Enlargement of right supraclavicular, bilateral hilar and mediastinal lymph nodes were also seen. Contrast-enhanced abdominal computed tomography showed accumulation of ascitic fluid and an enlarged lymph node (15 $\mathrm{mm}$ in diameter) inside the left common iliac artery. The left ovary was enlarged up to $50 \mathrm{~mm}$ in diameter.

Additionally, serum tumor maker tests were performed and levels of all three markers were elevated: carcinoembryonic antigen (CEA) $205.0 \mathrm{ng} / \mathrm{dL}$, carbohydrate antigen 125 (CA125) $372.9 \mathrm{U} / \mathrm{mL}$, and carbohydrate antigen 19-9 (CA 19-9) $2,888 \mathrm{U} / \mathrm{mL}$. Aspiration cytology cells of the right supraclavicular lymph node revealed metastatic adenocarcinoma (Fig. 2).

On the basis of these results, we diagnosed pulmonary lymphangitic carcinomatosis and in consideration of her age, we suspected ovarian cancer, or stomach cancer as her primary lesion. Subsequent pelvic magnetic resonance imaging revealed enlargement of left ovary with a fluid-fluid level. It did not have a solid pattern and appeared more like an endometrial cyst than ovarian cancer. Upper gastrointestinal endoscopy revealed no obvious abnormality. Colonoscopy could not be performed because of accumulation of feces.

Chemotherapy is not generally recommended for patients with such a poor respiratory condition, however, granting that the patient had no existing disease, she and her parents desired to receive aggressive therapy; thus we decided to start chemotherapy as an occult primary cancer. We chose carboplatin (AUC $=6$ ) and paclitaxel $\left(200 \mathrm{mg} / \mathrm{m}^{2}\right.$ ) as a regimen and started the chemotherapy one day after her admission. This regimen was determined with reference to the report from Briasoulis et al (7). Unfortunately however, her respiratory condition did not improve and she died of respiratory failure the day after the chemotherapy.

Autopsy, except for craniotomy, was performed $3 \mathrm{~h}$ after her death. Macroscopically, multiple white small nodules were observed in bilateral lungs and microscopically, lymph

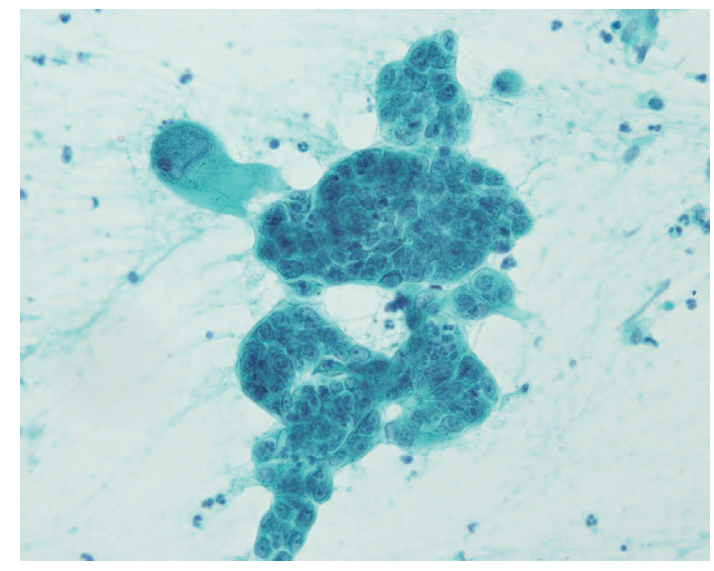

Figure 2. Aspiration cytology cells of the right supraclavicular lymph node showed adenocarcinoma.

vessels were filled with adenocarcinoma cells. The same microscopic findings were seen in some enlarged lymph nodes, including bilateral supraclavicular, bilateral hilar, mediastinal and intraabdominal lymph nodes (Fig. 3). A small polyp of $17 \times 10 \mathrm{~mm}$ was detected in the sigmoid colon. A loupe image showed a pedunculated polyp consisting of adenocarcinoma without an adenomatous component. It invaded the submucosal layer (Fig. 4). Microscopically, the tumor was composed of small clusters of neoplastic cells floating in clear spaces resembling lymphatic channels and invaded the serous membrane with marked lymphovascular infiltration. Immunohistochemically, the outer surface of the cell clusters was positive for MUC1, a characteristic 'inside-out' pattern for micropapillary carcinoma. In a part of the tumor, the spaces around the cell clusters were negative for D2-40 (Fig. 5). Based on the histological similarity to that of lung and other lymph node metastases, the final diagnosis was pulmonary lymphangitic carcinomatosis and multiple lymph node metastases of sigmoid colon cancer with micropapillary component (UICC-TMN classification: pT3N2M1, Stage IV).

\section{Discussion}

Since Siriaunkgul and Tavassoli (2) reported MC as a rare variant of invasive breast cancer in 1993, several MC cases had been reported in other organs. MC is a special type of adenocarcinoma with characteristic morphologic features and poorer prognosis regardless of anatomic location (8). There are some case reports on colorectal cancers with an $\mathrm{MC}$ component (8-12), but these are relatively rare. According to some available reports, the presence of MC component in colorectal cancer is likely to be associated with a poor prognosis as well as in other organs. Kim et al (13) reported that the presence of MC in the primary tumors was associated with more frequent lymphovascular invasion and lymph node metastases, a greater mean number of positive lymph nodes, and a higher tumor stage with more frequent distant metastases, compared with conventional adenocarcinoma. 

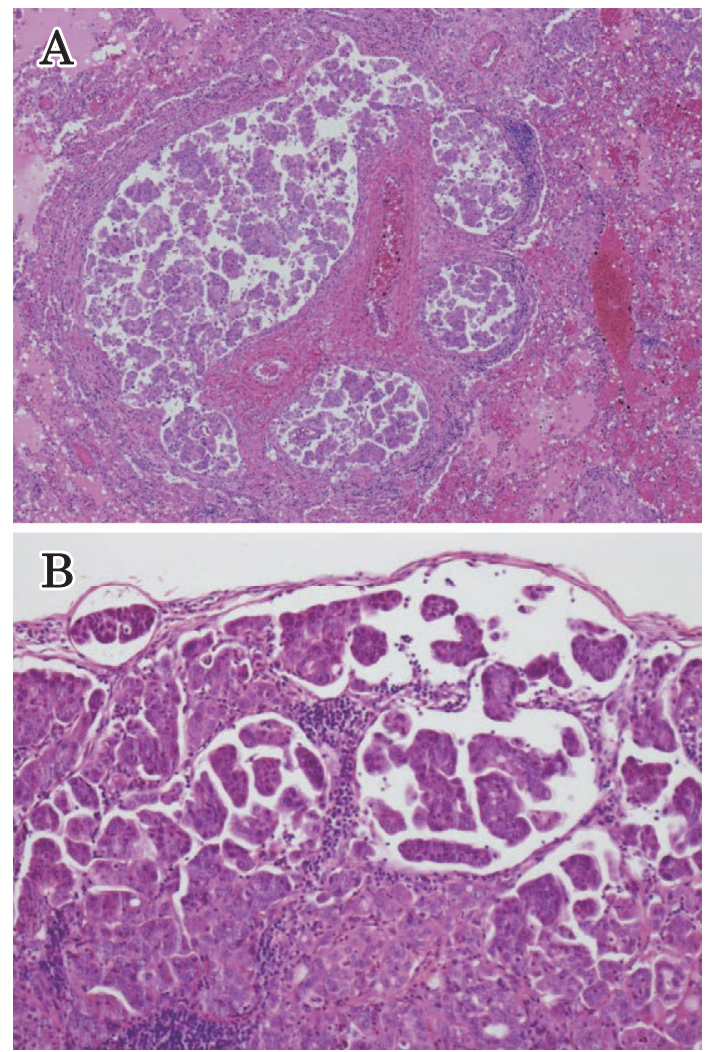

Figure 3. Microscopic findings of autopsy samples. (A) Lymph vessels in the lung were filled with adenocarcinoma cells and (B) the same finding was seen in the right supraclavicular lymph nodes.

Haupt et al (14) reported that the presence of micropapillary component was an independent predictor of regional nodal metastasis as well as tumor stage and lymphovascular invasion. The pathological feature of MC is characterized by immunostaining for MUC1, a glycoprotein typically expressed in the apical surface of normal epithelial cells, responsible for maintaining lumen formation (15). In MC, MUC1 expression is predominantly in the stroma-facing surface of the cell clusters and this characteristic 'inside-out' pattern is thought to cause the detachment of the neoplastic cells from the stroma, enhance their spread and lead to early dissemination to lymphovascular invasion. To distinguish the characteristic clear spaces around the tumor cell clusters from lymphatic channels, immunostaining for D2-40 is useful.

In the present patient, her disease began with respiratory symptoms. We made the diagnosis of pulmonary lymphangitic carcinomatosis with HRCT findings and aspiration cytology but could not identify the primary lesion until her autopsy was performed. We suspected several organs as a primary lesion but thought that she had a low potential for colon cancer. Yang and Lin (16) reported that carcinomas of the stomach, bronchus and breast were frequent primary lesions of pulmonary lymphangitic carcinomatosis, but colorectal cancer accounted for only $3.6 \%$. However, the cancer of this case was thought to have been at higher risk for pulmonary lymphangitic carcinomatosis than conventional ade-
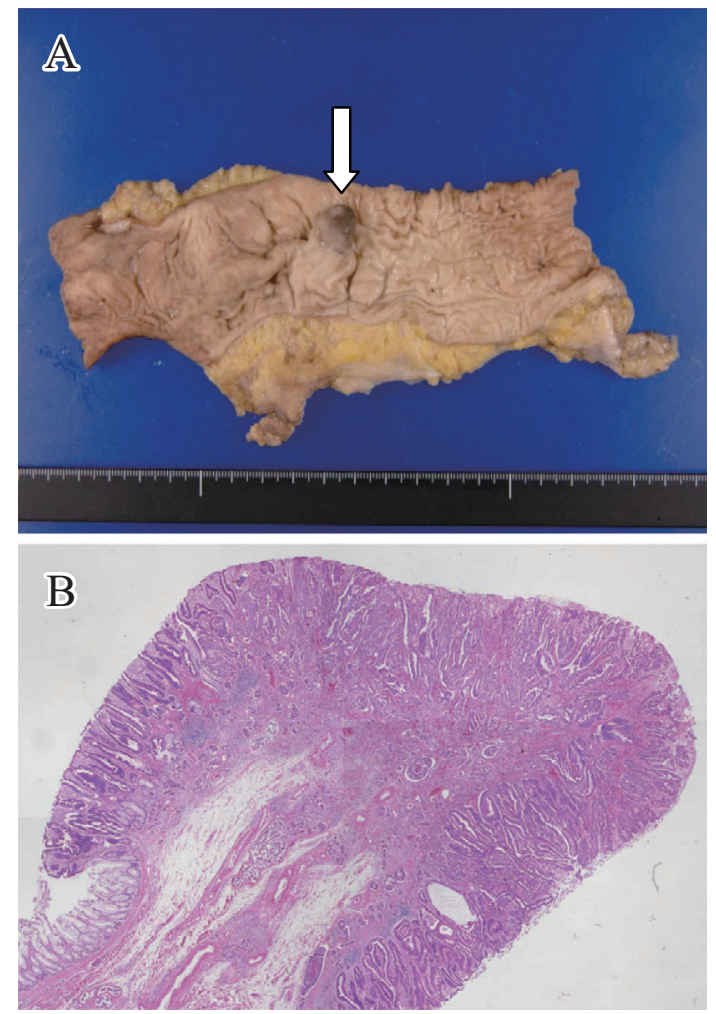

Figure 4. (A) Macroscopic findings of the sigmoid colon cancer (white arrowhead), measuring $17 \times 10 \mathrm{~mm}$. (B) A loupe image showed the pedunculated polyp consisting of adenocarcinoma.

nocarcinoma because of its pathological characteristic of high propensity for lymphovascular invasion.

Sato et al (17) retrospectively studied 1,011 patients with colorectal cancer, and clinicopathologically compared the patients under 40 years old with ones of 40 years and older. As a result, they reported that the percentage of female patients, lymphatic vessel invasion and node metastases had been significantly higher in the younger group. This result was also true of the present young patient. On the other hand, according to some reports $(13,14)$, colorectal micropapillary carcinoma does not have a tendency to develop in younger patients. Therefore, this patient was an extremely rare case in that colorectal cancer with MC component developed when she was only in her twenties.

In conclusion, we encountered a young patient of initial onset of pulmonary lymphangitic carcinomatosis caused by sigmoid colon cancer with the component of micropapillary carcinoma. To our knowledge, this is a rare case in that the disease occurred so early in life despite a lack of definite genetic predisposition and spread rapidly despite a smallness of primary lesion. This suggests that the cancer with MC component could occur in previously healthy young people and could spread through lymphatic vessels even if the primary lesion is still small. Therefore, one should consider a possibility of colorectal cancer with MC component if a pulmonary lymphangitic carcinomatosis with occult primary is detected. 

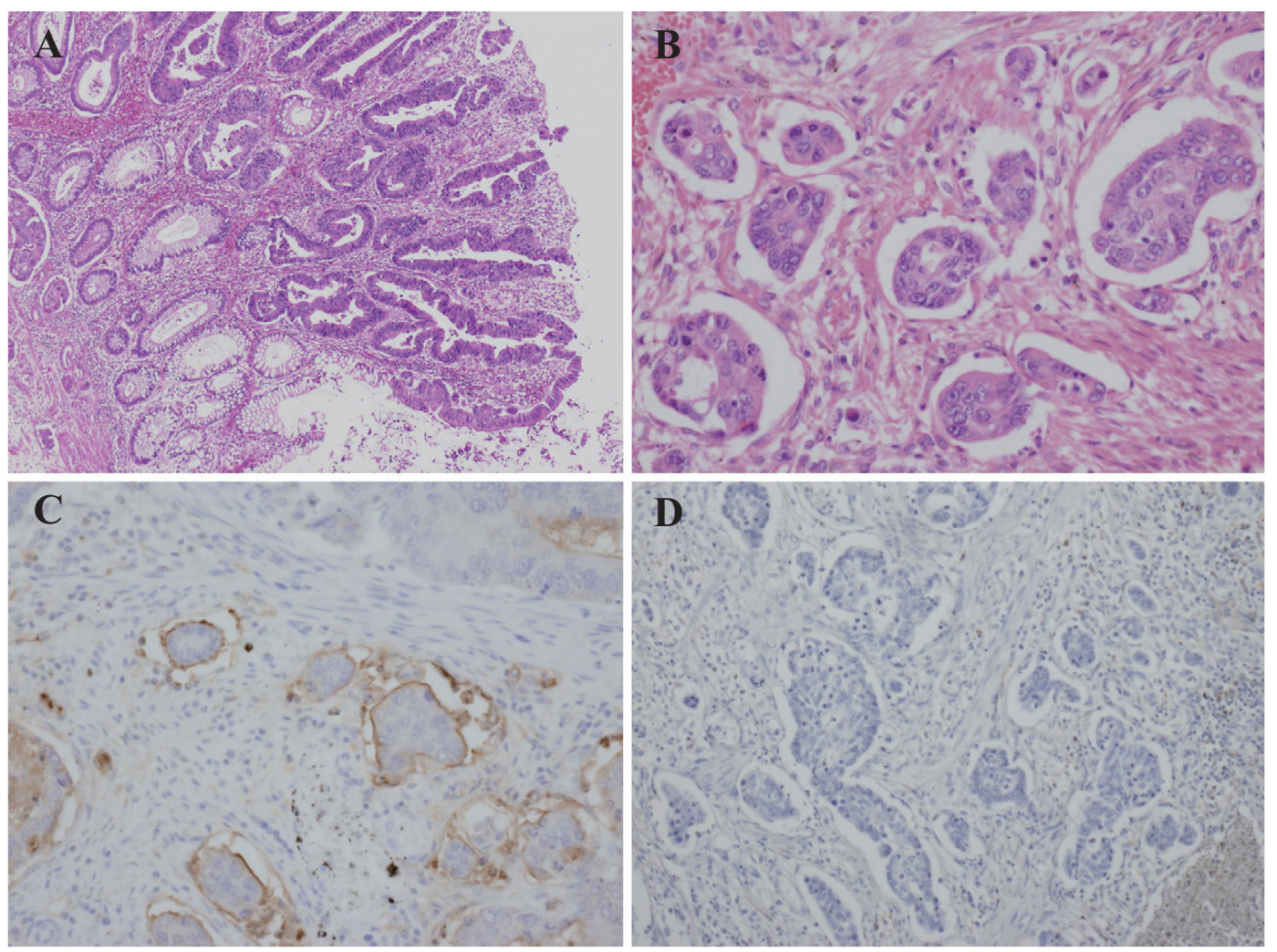

Figure 5. (A) Low-power view of sigmoid colon cancer. The tumor had no adenomatous component and it proliferated maintaining the tubular structure. The front formation was seen in the border area between tumor and normal epithelium. (B) High-power view of sigmoid colon cancer. The tumor was composed of small clusters of neoplastic cells floating in clear spaces resembling lymphatic channels. (C) MUC1 immunostaining of tumor cells. The outer surface of the cell clusters was positive for MUC1, so-called 'inside-out' pattern. (D) The spaces around the cell clusters were negative for D2-40 immunostaining and it suggested that these spaces were not lymphatic channels.

The authors state that they have no Conflict of Interest (COI).

\section{References}

1. Nassar H. Carcinomas with micropapillary morphology: clinical significance and current concepts. Adv Anat Pathol 11: 297-303, 2004.

2. Siriaunkgul S, Tavassoli FA. Invasive micropapillary carcinoma of the breast. Mod Pathol 6: 660-662, 1993.

3. Amin MB, Ro JY, El-Sharkawy T, et al. Micropapillary variant of transitional cell carcinoma of the urinary bladder. Histologic pattern resembling ovarian papillary serous carcinoma. Am J Surg Pathol 18: 1224-1232, 1994.

4. Amin MB, Tamboli P, Merchant SH, et al. Micropapillary component in lung adenocarcinoma: a distinctive histologic feature with possible prognostic significance. Am J Surg Pathol 26: 358-364, 2002.

5. Nagao T, Gaffey TA, Visscher DW, et al. Invasive micropapillary salivary duct carcinoma: a distinct histologic variant with biologic significance. Am J Surg Pathol 28: 319-326, 2004.

6. Gilks CB, Alkushi A, Yue JJ, Lanvin D, Ehlen TG, Miller DM. Advanced-stage serous borderline tumors of the ovary: clinicopathological study of 49 cases. Int J Gynocol Pathol 22: 29-36, 2003.

7. Briasoulis E, Kalofonos H, Bafaloukos D, et al. Carboplatin plus paclitaxel in unknown primary carcinoma: A Phase III Hellenic
Cooperative Oncology Group Study. J Clin Oncol 18: 3101-3107, 2000.

8. Wen P, Xu Y, Frankel WL, Shen R. Invasive micropapillary carcinoma of the sigmoid colon: distinct morphology and aggressive behavior. Int J Clin Exp Pathol 1: 457-460, 2008.

9. Sakamoto K, Watanabe M, De La, et al. Primary invasive micropapillary carcinoma of the colon. Histopathology 47: 479-484, 2005.

10. Hirota M, Yamashita K, Ichihara T, Nakamura E. A case of rectal cancer with the component of micropapillary carcinoma. Jpn J Gastroenterol Surg 41: 1741-1745, 2008.

11. Hisamori S, Nagayama S, Kita S, Kawamura J, Yoshizawa A, Sakai Y. Rapid progression of submucosal invasive micropapillary carcinoma of the colon in progressive systemic sclerosis: report of a case. Jpn J Clin Oncol 39: 399-405, 2009.

12. Sonoo H, Kameyama M, Inatugi N, Nonomura A, Enomoto $Y$. Pedunculated polyp of early sigmoid colon cancer with invasive micropapillary carcinoma. Jpn J Clin Oncol 39: 523-527, 2009.

13. Kim MJ, Hong SM, Jang SJ, et al. Invasive colorectal micropapillary carcinoma: an aggressive variant of adenocarcinoma. Hum Pathol 37: 809-815, 2006.

14. Haupt B, Ro JY, Schwartz MR, Shen SS. Colorectal adenocarcinoma with micropapillary pattern and its association with lymph node metastasis. Modern Pathol 20: 729-733, 2007.

15. Nassar H, Pansare V, Zhang H, et al. Pathogenesis of invasive micropapillary carcinoma: role of MUC1 glycoprotein. Mod Pathol 17: 1045-1050, 2004.

16. Yang SP, Lin CC. Lymphangitic carcinomatosis of the lungs. The 
Intern Med 50: 2361-2365, 2011 DOI: 10.2169/internalmedicine.50.5170

clinical significance of its roentogenologic classification. Chest $\mathbf{6 2}$ : 179-187, 1972.

17. Sato H, Maeda K, Hanai T, Koide Y, Funahashi M, Agata $T$. Clinicopathological study on patients less than 40 years old with colorectal cancer. Nihon Gekakei Rengo Gakkaishi (Journal of Japanese College of Surgeons) 34: 1-8, 2009 (in Japanese, Abstract in English).

(C) 2011 The Japanese Society of Internal Medicine http://www.naika.or.jp/imindex.html 